\title{
Apuntes sobre el léxico del poema VII de Catulo
}

\author{
Carolina Ponce HERnÁNDEZ \\ Universidad Nacional Autónoma de México
}

\begin{abstract}
A partir del carmen VII de Catulo, se ejemplifica la necesidad del conocimiento profundo del contexto y la cultura del autor de una obra literaria que debe poseer un traductor o estudioso de la literatura. Se evidencian las implicaciones que puede tener un solo vocablo en el sentido de un texto, ya que una sola palabra contiene en sí misma la intención del autor que la emplea, por lo que, a fin de comprender bien ese sentido, el traductor o estudioso de la literatura debe hacer una detallada investigación del contexto de la obra literaria y de la mentalidad del autor a estudiar o a traducir.
\end{abstract}

PALABRAS ClAVE: Catulo, literatura, léxico, traducción, Laserpicio.

In Catullus' VII carmen we can find an example of how it is necessary for a translator or literary critic to have a profound knowledge of an author's context and culture. It is also possible to exemplify the implications that the use of a certain word or term can have in the sense of a text; considering that a single word can contain in itself the author's intention. Therefore, in order to comprehend that sense, the translator or critic must elaborate a detailed investigation of the context in which the literary work was written and of the mentality of the author to be studied or translated.

KEY WORDS: Catullus, Literature, Lexicon, Translation, Laserwort.

La necesidad de consolidar el estudio de las lenguas como el fundamento esencial y fundamento ineludible de la cultura humana es casi tan obvio que parece darse por sentado. Sin embargo, cuando surgen ciertas postulaciones que pretenden establecer en las instituciones educativas la enseñanza de las lenguas como una disciplina que surge por generación espontánea y cuyas venas no están repletas de la sangre que proporciona el contexto cultural, es preciso señalar con toda claridad que el conocimiento de una lengua, cualquiera que sea, está necesariamente alimentado por todo lo que el hombre que la posee y emplea ha hecho y ha dicho a lo largo de su historia.

Puesto que sería muy tedioso insistir sobre lo anterior, he considerado que un ejemplo puede ser mucho más explícito. Ya Séneca decía verba monent exempla trahunt; por lo tanto vayan unas breves observaciones en torno a un también breve poema para tratar de convencer con esos argumentos de que es preciso estudiar las lenguas y sus 
culturas conjuntamente, de donde deriva el término compuesto, yuxtapuesto de lenguas-culturas.

Quaeris, quot mihi basiationes tuae, Lesbia, sint satis superque. quam magnus numerus Libyssae harenae lasarpiciferis iacet Cyrenis oraclum Iouis inter aestuosi et Batti ueteris sacrum sepulcrum; aut quam sidera multa, cum tacet nox, furtiuos hominum uident amores: tam te basia multa basiare uesano satis et super Catullo est, quae nec pernumerare curiosi possint nec mala fascinare lingua.
Preguntas, Lesbia, cuántos besos tuyos

Me serían bastantes y de sobra.

Tantos como la arena de Libia que

Que yace en la laserpecífera Cirene entre el oráculo de Júpiter ardiente

y el sagrado sepulcro del viejo Bato

o cómo las muchas estrellas que, cuando calla

la noche,

ven los amores furtivos de los hombres

que tú beses muchos besos

es bastante y de sobra para el loco Catulo

los que ni puedan contar los curiosos, ni hechizar una mala lengua.*

(Catulo, VII ad Lesbiam)

En los versos anteriores Catulo, el famoso poeta lírico del siglo I a. C., emplea un léxico perfectamente escogido para expresar el deseo apasionado, que raya en la locura, de ser besado por su amada Lesbia.

Lo primero que hacemos es descubrir en el poema una especie de diálogo centrado en el tema del amor y expresado con el lugar argumentativo de los besos. El breve texto de doce versos nos introduce en el deseo y los goces de amores furtivos, en un lugar caliente (aestuosi), cuando la noche es iluminada por los astros.

Este poema está íntimamente relacionado con otro de Catulo (V), en el que le pedía a su amada Lesbia tres mil trescientos besos, tantos que no pudieran contarse. ${ }^{1}$ Sin embargo, después de la primera lectura, cuya impresión deja, sin duda, su sello emotivo-estético en la memoria, es preciso abundar y encontrar en el poema las razones que lo llevan a ser juzgado como un modelo: ¿por qué si parece tan común, puede despertar sensaciones, provocar opiniones y conturbar?

El análisis de esas razones conducen al lector, al traductor, al investigador de la literatura y de la poética por muchos caminos: la retórica, la métrica, la poesía erótica latina, la construcción epigramática, etcétera. En este estudio deseo revisar sólo algunas cuestiones relativas al léxico y cruzar informaciones sobre cierto término que emplea el poeta, con objeto de entender un poco más la excelencia de sus versos.

Un traductor que simplemente llegara a realizar una primera versión del texto, sin duda no lo entendería de manera cabal y por lo tanto no podría transmitir el contenido pleno del mensaje; en consecuencia, no se lograría la comunicación entre el autor, el

* Todas la traducciones son mías.

1 "Vivamus mea Lesbia, atque amemus,/ rumoresque senum seueriorum/ omnes unius aestimemus assis!/ soles occidere et redire possunt:/ nobis cum semel occidit breuis lux,/ nox est perpetua una dormienda./ da mi basia mille, deinde centum,/ dein mille altera, dein secunda centum,/ deinde usque altera mille, deinde centum./ dein, cum milia multa fecerimus,/ conturbabimus illa, ne sciamus,/ aut ne quis malus inuidere possit,/ cum tantum sciat esse basiorum" (Catulo, V ad Lesbiam). 
traductor y el receptor del poema. La primera parte nos conduce a un tópico usado miles de veces en la literatura lírica amorosa, esto es, la pregunta que una mujer, Lesbia, le hace al hombre que la ama sobre cuántos besos son necesarios para satisfacer el deseo del amante. Con base en la hipérbole presente en el número de granos de arena y de estrellas, el poeta se desplaza del plano terrestre a la esfera universal, y demuestra de forma unificadora cómo el amor que surge y se nutre de los sentidos es capaz de llevar a los amantes a los más completos goces. En un sentido semejante, indica de forma indirecta pero alusiva que la relación amorosa se establece en un lugar divino y sagrado que va del ámbito de una figura preponderante, el mítico Bato, hasta el mismo Júpiter. Llegando a ese punto, ni el traductor ni el lector de la traducción tendrían mayores problemas.

Pero más allá de la hipérbole, a lo largo de los versos, el lector del latín, el traductor a la lengua española y el lector de la traducción se encuentran con múltiples problemas para entender a fondo el contenido y por lo tanto desentrañar el mensaje y lograr que se establezca la comunicación plena.

En primer lugar, el término Libyssae, que corresponde al adjetivo para designar lo procedente de Libia, la conocida región situada en el norte de África. En segundo lugar, el adjetivo lasarpiciferis, que significa laserpicífera, para hablar de Cirene, y que el poeta emplea para calificarla. Pero ¿qué significa laserpicífera y qué es Cirene? En cuanto al nombre propio Cirene sabemos que es aquella ciudad de Libia que en las épocas antiguas fue importante, entre otras cosas, por dos razones: haber sido la patria del poeta Calímaco y haber sido gran productora de una planta llamada laserpicio. Del sustantivo laserpicio se deriva el adjetivo laserpicífera del cual el diccionario nos informa que significa que es productora de dicha planta.

Sin embargo, ¿qué es el laserpicio? Para resolver esto, el lector del texto latino y el traductor tienen que realizar una amplia investigación y encontrar en primer lugar su etimología. Al respecto, Alfred C. Andrews (232-236) nos informa que el término laserpicio corresponde al griego $\sigma \imath \lambda \varphi \imath o ́ v$, procede del jónico y parece que no tiene fuente indoeuropea, sino que sea tal vez cirenaica; de allí procedería la parte sirpe del latín lasserpicium, al que se habría añadido con síncopa el prefijo indoeuropeo lac (leche), para significar el laser que era el jugo lechoso que exudaba el sirpe. Este sirpe, a su vez, contiene en su última sílaba el sustrato pix que sirve para designar la brea (en inglés pitch), por tener el jugo en estado gomoso o resinoso. Tendríamos pues esta formación: lac-sir $>$ lasser-pic $<[p i x]-i$-um. Otra información al respecto de su etimología dice que la forma sirpe llega al latín a través del etrusco (Corti).

En segundo lugar, derivado del anterior, está seguir la historia textual de la palabra, lo que sin duda extendería más allá de lo conveniente este trabajo; por lo que señalaremos sólo algunos autores importantes que la han empleado, bajo la consideración de que la mayoría son filósofos de la ciencia en el sentido de la phylosophia naturalis, es decir, en este caso, estudiosos de la medicina y de las plantas y sus localizaciones y usos. Los autores griegos, como es lógico, emplean generalmente silfión más que laserpicio, 
y así lo encontramos en Aristófanes ${ }^{2}$ (Andrews: 234; Riddle: 8), Heródoto ${ }^{3}$ (Andrews: 233), Hipócrates ${ }^{4}$ (ibid.), Teofrasto ${ }^{5}$ (Andrews: 233; Riddle: 8), Dioscórides ${ }^{6}$ (Andrews: 233; Riddle: 9) y Galeno7 (Andrews: 233; Riddle: 9).

Entre los autores latinos que hablan del laserpicio están Columela ${ }^{8}$ (Andrews: 233), Plinio y Sorano ${ }^{9}$ (Riddle: 7), el más famoso ginecólogo de la época de Trajano y Adriano, todos ellos posteriores a Catulo.

Es evidente que el silfio o laserpicio era conocido desde el siglo vi a. C., puesto que está documentado tanto en los escritos como en la cerámica y poco después en las monedas, específicamente en las monedas de Cirene, las cuales contenían en uno de sus lados la representación de la planta en manojo o gavilla como símbolo de la ciudad, puesto que era sin duda la fuente principal de su riqueza agrícola y comercial.

La historia de las palabras silfio y laserpicio a lo largo de los tiempos hasta nuestros días, ha dado lugar a un sinnúmero de fichas, cédulas, entradas y tratados sobre sus aplicaciones, encontrándose un interés muy focalizado en su uso como anticonceptivo y abortivo.

Expuesta la formación de la palabra, ya sea en forma simple o compuesta como silfio o laserpicio, y el empleo que de ella hicieron los antiguos, veamos ahora qué es el laserpicio como planta. El laserpicio es una planta nativa del norte de África, en forma de arbusto que desde la antigüedad y hasta nuestros días, en diferentes especies que evolucionaron y derivaron de la especie antigua, ha servido como base para producir condimentos, estimulantes y remedios ${ }^{10}$ para las enfermedades respiratorias, de los sistemas nervioso, digestivo y urinario, y que también se empleaba como anticonceptivo y abortivo, ${ }^{11}$ según hemos dicho, lo que nos lleva a plantear una relación por lo menos alusiva, cuando lo encontramos en un poema erótico. Esta planta pertenece al orden de las apiales, familia de las apiaceae, género ferula, especie $f$. assafoetida, ${ }^{12}$ denominada en castellano como asafétida. La especie actual es una planta herbácea y perenne que puede crecer hasta dos metros de altura, cuyo tronco es de cinco a

${ }^{2}$ Vid. Aristófanes. Plut. 925 ad Schol. Ad I.

${ }^{3} \mathrm{Vid}$. Heródoto. IV.149.

${ }^{4}$ Vid. Hipócrates. Acut. 387, 389.

${ }^{5}$ La obra de Teofrasto que estudian los investigadores es De historia et causa plantarum, VI. 3.

${ }^{6}$ Vid. Discórides. De materia medica. III, 48, 80, 81, 83 y 84.

${ }^{7}$ Vid. Galen. Comm. in Hippocr. De victu acut. 4.877, donde asegura que el Silfión fue originalmente el nombre para la raíz y que después gradualmente se uso para toda la planta. Galen, De simplicium medicamentorum termperamentis ac facultatibus. VIII. 16.4.

${ }^{8}$ Vid. Colum. De re rustica 12.7.7; 7.17.7.

${ }^{9} \mathrm{Vid}$. Soranus. Gynaeciorum libri. IV, i. 60, 63.

${ }^{10} \mathrm{Cf}$. Ferula Assafoetida en http://es.wikipedia.org/wiki/Ferula_assafoetida; Silfio, en http://www.summagallicana.it/lessico/s/silfio.htm; Silfio; http://es.wikipedia.org/wiki/Silfio; Silphium laciniatum en http:// www.homeopatiageneral.com/silphium-laciniatum.html. Fecha de acceso a las páginas anteriores: 1 de febrero de 2013.

${ }^{11}$ Cf. Johnston: 328-329; Moorhouse: 417-418; Andrews y Riddle.

${ }^{12}$ Sobre las discusiones en torno al silfio y si es o no una ferula assafoetida, además de las confusiones de Teofrastro que continuaron en toda la literatura posterior, $c f$. Lykoudis. 
ocho centímetros de diámetro en la base; las hojas tienen una longitud de treinta a cuarenta centímetros, y su flor es amarilla. Sus hojas de color dorado caían con el viento dominante del sur, en el periodo de ascensión de la estrella del can, es decir, durante la canícula (vid. "Silfio", Wikipedia). Fue descrita por Carlos Lineo y aparece publicada su taxonomía en Species plantarum 1:248 (1753).

Para nosotros, es importante señalar que Catulo parece ser el primero que acuña el adjetivo laserpicífero, esto es, nos encontramos ante un apax o neologismo inventado por el poeta. El neologismo del poeta nos conduce a indagar qué causas motivan su acuñación, pues no satisface la fácil respuesta de que necesitaba un número determinado de sílabas largas y breves para lograr la construcción de su metro faleuco o falecio (- - - u u - u - u - $\underline{u})$, el endecasílabo preferido de Catulo, metro en que está escrito el poema.

Sin duda la creación del término fue resultado de pensar y considerar las ricas connotaciones que proporcionaba a su poema, connotaciones que el estudioso lector o traductor debe rastrear en los campos de la cultura no sólo antigua sino también la actual. Con lo que constatamos de nuevo la necesaria preparación que como especialistas en las lenguas y sus literaturas debemos adquirir, a fin de comprenderlas de manera cabal.

Sigamos con nuestro poeta y su neologismo. De acuerdo con Plinio el Viejo (23-79 d. C.), quien vivió alrededor de ciento treinta años después de la muerte de Catulo, ${ }^{13}$ sabemos que el laserpicio se encontraba en la provincia de Cirene, que de esa planta

${ }^{13} 15$. Ab his proximum dicetur auctoritate clarissimum laserpicium, quod Graeci silphion vocant, in Cyrenaica provincia repertum, cuius sucus laser vocatur, magnificum in usu medicamentisque et ad pondus argentei denarii repensum. [39] multis iam annis in ea terra non invenitur, quoniam publicani, qui pascua conducunt, maius ita lucrum sentientes depopulantur pecorum pabulo. unus omnino caulis nostra memoria repertus Neroni principi missus est. si quando incidit pecus in spem nascentis, hoc deprehenditur signo: ove, cum comederit, dormiente protinus, capra sternuente crebrius. [40] diuque iam non aliud ad nos invehitur laser, quam quod in Perside aut Media et Armenia nascitur large, sed multo infra Cyrenaicum, id quoque adulteratum cummi aut sacopenio aut faba fracta; quo minus omittendum videtur C. Valerio $\mathrm{M}$. Herennio cos. Cyrenis advecta Romam publice laserpicii pondo XXX, Caesarem vero dictatorem initio belli civilis inter aurum argentumque protulisse ex aerario laserpicii pondo MD.

[41] Id apud auctores Graeciae [c]e[r]tissimos invenimus natum imbre piceo repente madefacta tellure circa Hesperidum hortos Syrtimque maiorem septem annis ante oppidum Cyrenarum, quod conditum est urbis nostrae anno CXLIII, vim autem illam per IIIIstadium Africae valuisse. [42] in ea laserpicium gigni solitum, rem feram ac contumacem et, si coleretur, in deserta fugientem, radice multa crassaque, caule ferulaceo ac simili crassitudine. huius folia maspetum vocabant, apio maxime similia. semen erat foliaceum, folium ipsum vere deciduum. [43] vesci pecora solita primoque purgari, mox pinguescere, carne mirabilem in modum iucunda. post folia amissa caule ipso et homines vescebantur mo[d]is omnibus decocto, elixo assoque, eorum quoque corpora XL primis diebus purgante. sucus duobus modis capiebatur, e radice atque caule, et haec duo erant nomina, $\mathrm{r}[\mathrm{h}$ ]izias atque caulias, vilior illo ac putrescens. [44]radici cortex niger. ad mercis adulteria sucum ipsum in vasa coiectum admixto furfure subinde concutiendo ad maturitatem perducebant, ni ita fecissent, putrescentem. argumentum erat maturitatis colos siccitasque sudore finito. [45] alii tradunt laserpicii radicem fuisse maiorem cubitali, tuberque in ea supra terram. hoc inciso profluere solitum sucum ceu lactis, supernato caule, quem magydarim vocarunt. folia aurei coloris pro semine fuisse, cadentia a canis ortu austro flante. ex his laserpicium nasci solitum, annuo spatio et radice et caule consum[m]antibus 
se extraía un jugo llamado laser de gran valor. Pero Plinio señala que en su siglo ya no se encontraba el laserpicio de Cirene, porque se había extinguido por dos causas: el ganado que se alimentaba de dicho arbusto y la excesiva devastación que los hombres que comerciaban con él habían realizado. Plinio incluso informa que el último ejemplar registrado fue uno enviado a Nerón, y que antes, siendo ya César dictador (c. 46 a. C.), ordenó que se pagaran treinta libras de laserpicio cirenaico con la exorbitante cifra de mil quinientas libras romanas de oro y plata.

En época de Plinio, otro laserpicio, de menor calidad (multo infra cyrenaicum), era producido en tierras de Persia, Media y Armenia. Entre las propiedades ${ }^{14}$ que se adju-

sese. hi et circumfodi solitum prodidere, nec purgari pecora, sed aegra sanari aut protinus mori, quod in paucis accidere. Persico silphio prior opinio congruit (Plin. Naturalis Historia. XIX. 15 [38]).

${ }^{14}$ Imbribus proveniunt omnia haec, imbre et silphium venit primo, ut dictum est. ex Syria nunc hoc maxime inportatur, deterius Parthico, sed Medico melius, extincto omni Cyrenaico, ut diximus. usus silphii in medicina foliorum ad purgandas vulvas pellendosque emortuos partus; decocuntur in vino albo et odorato, ut bibatur mensura acetabuli a balineis. radix prodest arteriis exasperatis, collectionibus sanguinis inlinitur. sed in cibis concoquitur aegre, inflationes facit et ructus. urinae quoque noxia e[st], suggillatis cum vino et oleo amicissima et cum cera strumis. verrucae sedis crebriore eius suffitu cadunt. 49. [101] Laser e silphio profluens quo diximus modo inter eximia naturae dona numeratum plurimis compositionibus inseritur, per se autem algores excalfacit, potum nervorum vitia extenuat. feminis datur in vino et lanis mollibus admovetur vulvae ad menses ciendos. pedum clavos circumscariphatos ferro mixtum cerae extrahit. urinam ciet ciceris magnitudine dilutum. [102] Andr[e]as spondet copiosius sumptum nec inflationes facere et concoctioni plurimum conferre senibus et feminis, item hieme quam aestate utilius, et tum aquam bibentibus. cavendum, ne qua sit exulceratio intus. ab aegritudine recreationi efficax in cibo; tempestive enim datum cauteri[i] v[i]m optinet, adsuetis etiam utilius quam expertibus. [103] extera corporum indubitatas confessiones habent: venena telorum et serpentium extinguit potum; ex aqua vulneribus his circumlinitur, scorpionum tantum plagis ex oleo, ulceribus vero non maturescentibus cum farina hordeacia vel fico sicca, carbunculis cum ruta vel cum melle vel per se visco superlitum, ut haereat; sic [e]t ad canis morsus; excrescentibus circa se[d]em cum [t]egmine punici mali ex aceto decoctum, clavis, qui volgo morticini appellantur, nitro mixto. [104] alopecias nitro ante subactas replet cum vino et croco aut pipere ac murium fimo et aceto. perniones ex vino fovet et ex oleo coctum; inponitur sic et callo, clavis pedum superrasis; praecipuae utilitatis contra aquas malas, pestilentes tractus vel dies; in tussi, uva; fellis veteri suffusion $<\mathrm{i}>$, hydropicis, raucitatibus; confestim enim purgat fauces vocemque reddit. [105]podagras in spongea dilutum posca lenit. pleuriticis in sorbitione vinum poturis datur, contractionibus, opisthotonicis ciceris magnitudine cera circumlitum. in angina gargarizatur. anhelatoribus et in tussi vetusta cum porro ex aceto datur; $\mathrm{a}<\mathrm{t}>$ ex aceto his, qui coagulum lactis sorbuerint, praecordiorum vitiis syntecticis, comitialibus cum vino, in aqua mulsa linguae paralysi. coxendicibus et lumborum doloribus cum decocto melle inlinitur. [106] non censuerim, quod auctores suadent, cavernis dentium in dolore inditum cera includi, magno experimento hominis, qui se ea de causa praecipitavit ex alto. quippe tauros inflammat naribus inlitis, serpentes avidissimas vini admixtum rumpit; ideo nec inungui suaserim, cum Attico licet melle praecipiant. quas habeat utilitates admixtum aliis, inmensum est referre, et nos simplicia tractamus, quoniam in his naturam esse apparet, in illis coniecturam saepius fallacem, nulli satis custodita in mixturis concordia naturae ac repugnantia. qua de re mox plura. 50. [107] Non esset mellis auctoritas in pretio minor quam laseris, ni ubique nasceretur. illud ipsa fabricata $<\mathrm{e}>$ st natura, sed huic gignendo animal, ut diximus, innumeros ad usus, si quotiens misceatur aestimemus. prima propolis alvorum, de qua diximus, aculeos et omnia infixa corpori extrahit, tubera discutit, dura concoquit, dolores nervorum mulcet ulceraque iam desperantia cicatricem cludit. [108] mellis quidem ipsius natura talis est, ut putrescere corpora non sinat. iucundo sapore atque non aspero, alia quam salis natura, faucibus, tonsillis, anginae omnibusque oris desideriis utilissimum arescentique in febribus linguae, iam vero peripneumonicis, pleuriticis decoctum, item vulneribus, a serpente percussis et contra 
dicaban a la planta estaban: para el ganado servía primero como un purgante, después lo engordaba haciendo que su carne fuera admirablemente agradable. Como medicina Plinio dice que la raíz curaba las arterias inflamadas; que el jugo de la raíz y del tallo servía de purgante; cocinado con la comida producía inflamación y era dañino para la orina (sic); provocaba la caída de las verrugas; quitaba la fiebre y calmaba o reanimaba los nervios; mezclado con cera servía para extraer clavos enterrados en los pies; era antídoto para el veneno de flechas, serpientes y alacranes; en polvo mezclado con otras sustancias secaba las úlceras; ayudaba a quitar la tos, la ronquera, la hidropesía, la podagra; en concentrado de vinagre, mezclado con vino, regularizaba el ritmo cardiaco; para las mujeres, el jugo de las hojas servía para limpiar la matriz y la vulva expulsando todo residuo de un aborto; cuando se daba a las mujeres en vino o se aplicaba en vendas suaves se provocaba la menstruación.

Hemos resumido el texto de Plinio, pero el lector interesado podrá revisarlo directamente y encontrar en él todo un apartado informativo sobre los usos medicinales del laserpicio, acercándose a una ventana extraordinaria que nos permite ver un cuadro de la ciencia antigua, donde la mezcla de partes de la planta con sustancias derivadas de la misma con vino, agua, miel, azufre, etcétera, daba por resultado medicamentos distintos para diferentes enfermedades, así como también el uso de cada una de las partes de la planta servía como cura para diversas enfermedades, señalando para qué se utilizaban la raíz, la corteza de la raíz, el tallo, las hojas antes de desprenderse del tallo y las que ya habían caído; cuál era el uso de ella transformada en aceite, vinagre, polvo, etcétera. ${ }^{15}$

En este punto no podemos dejar de imaginar que Lesbia, haya sido Claudia Pulchra u otra, Sempronia o Cintia, Corina, Sulpicia y muchas mujeres romanas conocieron el laserpicio y lo emplearon como preventivo para evitar el embarazo, puesto que provocaba la menstruación y se indicaba contra la dismenorrea, o como abortivo y purificador, después del aborto o del parto, de los genitales femeninos.

Ahora bien, ante ese escenario de la farmacopea de la antigüedad era lógico que tan amplia utilización del laserpicio y su alto valor económico fueron claves para que Catulo acuñara el adjetivo laserpicífero porque así como la arena de Cirene produce y sostiene la planta curativa (relacionada con los ciclos femeninos y la interrupción del embarazo), así también los incontables besos de Lesbia producirán la satisfacción amorosa del loco Catulo (vesano Catullo). Y es esa locura la que conlleva las hipérboles antes mencionadas del poema; pero, como dijo Plinio, la planta se usaba también

venena, fungos, paralytic $<i>s$ in mulso, quamquam suae mulso dotes constant. mel auribus instillatur cum rosaceo, lendes et foeda capitis animalia necat. [109] usus despumati semper aptior, stomachum tamen inflat, bilem auget, fastidium creat. et oculis per se inutile aliqui arbitrantur; rursus quidam angulos exulceratos melle tangi suadent. mellis causas atque differentias nationesque et $\mathrm{i}<\mathrm{n}>$ dicationem in apium ac deinde florum natura diximus, cum ratio operis dividi cogeret miscenda rursus naturam rerum pernoscere volentibus (Plin. Op. cit. XXII 48, 49. [ 100, 109]).

${ }^{15} \mathrm{Cf}$. Riddle. En este artículo se presentan ejemplos del uso y los experimentos médicos que en la actualidad se realizan con las plantas que pertenecen a la familia del laserpicio cirenaico antiguo. 
para calmar los nervios, para atenuar los disturbios del sistema nervioso y además para regularizar el ritmo cardiaco, dos aspectos que se reflejan en el poema a través del uso de repeticiones, derivaciones y el tipo de léxico elegido por el poeta. Esto se ve muy claramente en basiationes derivado del verbo basiare, a su vez derivado de basia, en donde basiationes es la acción de besar, basiare es besar y basia son los besos, expuestos de una manera explicita desdoblando el acusativo interno del verbo; este acusativo está presente en el mismo verso- besar besos, que tú beses muchos besos (Cf. Bertman: 477-478). También encontramos otra repetición a distancia de los sinónimos satis et super - bastante y más-; o el uso de magnus y multa, este último repetido; o el sustantivo numerus y su verbo derivado pernumerare, en donde el numerus es magnus - un gran número- y el verbo pernumerare es intensificativo y perfectivo por el prefijo per.

Con todas esas repeticiones se producen efectos fonéticos que le dan al poema en lengua latina un sonido aliterativo que puede reproducir perfectamente el ritmo del corazón del poeta y el estado nervioso (vesano) en que se encuentra.

En cuanto a los siguientes versos que hablan del oráculo de Júpiter ardiente y el sepulcro del viejo Bato, el lector y el traductor tienen también que hacer un esfuerzo de imaginación, que sólo se verá completado plenamente si el traductor realiza una investigación y el lector tiene frente a sí una buena edición con las notas que le expliquen cada uno de los puntos. En esta parte sólo mencionaré que Bato es un epíteto patronímico que se refiere a Calímaco como una especie de descendiente del tal Bato, y que nos informa que ahí estaba posiblemente la tumba de Calímaco. Por una parte, Bato, de acuerdo con una tradición de la Libia (Bertman: 477), era el nombre del mítico fundador de Cirene, pero había recibido ese nombre porque tenía un defecto al hablar, pues en griego $\beta \alpha \tau \tau \alpha \rho i ́ \zeta \omega$ significa tartamudear, y es una palabra que pertenece al campo semántico de la oralidad y de la boca, como sucede con todos los derivados de basia que vimos antes, pero también relacionada con la palabra oraclum que literalmente significa boca pequeña, y que está planteada ahí como el oráculo de Júpiter y más aún con el vocablo lingua que cierra todo el poema en una peroración que le sirve al poeta para exponer su deseo de que los besos no sean hechizados por una mala lengua. Pero de lo anterior se desprenden todavía una serie de interrogantes, la principal ¿quién fue Calímaco?, y de ella ¿por qué Calímaco es importante para Catulo?, al punto de hacer dos referencias a él en un poema amoroso, esto es Cirene y Bato.

No contestaré ampliamente lo anterior, porque entrar al estudio de Calímaco es una labor ingente; sin embargo, de manera muy breve, señalaré sólo que fue el encargado de dirigir la gran biblioteca de Alejandría, uno de los grandes filólogos de la antigüedad y un poeta original e innovador que marcó senderos representativos de la cultura helenística. Como poeta produjo pequeñas obras líricas que fueron modelo de Catulo.

Hemos visto como a través de un poema lírico de carácter amoroso llegamos a tener un esbozo del mundo antiguo. Naturalmente un análisis más detallado con investigaciones más enriquecidas en torno al vocabulario, el metro y las referencias que contiene podrían conducir al traductor y al lector a una comprensión cada vez más cabal y 
profunda de la obra, del autor y del mundo en que se gestó. Por lo cual quede todo ello como un ejemplo de que para entender absolutamente una obra literaria, por pequeña que sea, se requiere de una acuciosa labor lingüística, histórica, mitológica, geográfica, etcétera, es decir, de una detallada labor filológica.

Así pues, el estudio de las lenguas y los textos que se han expresado en ellas sólo puede ser hecho de manera adecuada si partimos de la indispensable necesidad de conocer la cultura, esto es el contexto en el que se entiende el mensaje completamente. Por lo tanto, las carreras de letras en esta Universidad Nacional Autónoma de México deben obligatoriamente estudiarse teniendo como fundamentos los aspectos históricos en su más amplia comprensión; los aspectos correspondientes a la filosofía y las ideologías; los aspectos que relacionan los textos literarios con las otras artes, y sin lugar a dudas, todos los aspectos lingüísticos que atienden a la comprensión completa de los contenidos, pues sólo de esa forma se establece la comunicación efectiva y creativa, capaz de elaborar nuevos conocimientos y seguir construyendo lo mejor de la cultura humanística.

Ahora bien, es primordial no perder de vista que nuestra universidad es nacional, y por tal carácter tiene la responsabilidad de ofrecer en los campos científico, humanístico y social, las carreras y disciplinas de estudio que no están cubiertas en otras instituciones de educación superior, y éste es precisamente el caso de casi todas las carreras de letras. Ello significa que todos aquellos mexicanos que tengan el interés de estudiar y ser profesionales bien preparados, productivos y creativos en las letras clásicas o las letras italianas o las letras alemanas, etcétera, tienen como único lugar que les puede proporcionar tal preparación la Universidad Nacional Autónoma de México.

Está de más decir que más allá del panorama nacional, nuestra Universidad es también el centro al que recurren los interesados de otras naciones latinoamericanas, e incluso de países de otros continentes.

En consecuencia, por muchas razones, las carreras de letras de la UNAM deben tender a ser cada vez más profundas, más críticas, más amplias, con el fin de cumplir cabalmente con su responsabilidad social y humanística, y de ninguna manera puede aceptarse que dirijan su curso a enseñanzas superficiales, simplonas y baladíes.

\section{Obras citadas}

ANDREWS, Alfred. "The Silphium of Ancients: A lesson on Crop Central". Isis, vol. 33, núm. 2. The University of Chicago Press on Behalf of The History of Science Society. Pp. 232-236. 14 de febrero de 2013. <http://www.jstor.org/ stable/330743>.

BerTMAn, Stephen. 1978. "Oral Imagery in Catullus 7". The Classical Quarterly, vol. 28, núm. 2. New Series. Cambridge: Cambridge University Press. Pp. 477-478. 14 de febrero de 2013. <http://www.jstor.org/stable/638704>.

Catulo. "Catullus". The Latin Library. 15 de febrero de 2013. <http://www.thelatinlibrary.com/catullus.shtml>. 
Cayo Plinio Segundo. 1905. "Naturalis historia". Bibliotheca Augustana. Ed. L. IAN y C. MAYHOFF. Leipzig. 14 de febrero de 2013. <http://www.hs-augsburg. de/ harsch/Chronologia/Lspost01/PliniusMaior/plm_hi19.html>.

CORTI, Elio. "Silfio, cantaride Lytta vesicatoria". Suma Gallicana. 14 de febrero de 2013. <www.summagallicana.it/lessico/s/silfio.htm>.

Johnston, Patricia. 1993. "Love and Laserpicium in Catullus 7". Classical Philology, vol. 88, núm. 4. Chicago: The University of Chicago Press. Pp. 328329. 18 febrero de 2013. <http://www.jstor.org/stable/270217>.

LinNAeI, Caroli.1753. Species Plantarum, vol. 1. Holmiae, Laurentii Salvii, 560 $\mathrm{pp}$.

LyKOUDIS, María. "In Search of Silphion". 13 de febrero de 2013. <http://www. hort.purdue.edu/newcrop/hort_306/reading/Reading\%2017-1.pdf > .

Moorhouse, A. C. 1963. "Two Adjectives in Catullus 7". The American Journal of Philology, vol. 84, núm. 4. Baltimore: The Johns Hopkins University Press. Pp. 417-418. 14 de febrero de 2013. <http://www.jstor.org/stable/293237>.

RidDLE, John. 1991. "Oral contraceptives and Early-Term Abortifacients during Classical Antiquity and the Middle Ages". Past and Present, vol. 132, núm. 1. Oxford: Oxford University Press. Pp. 3-32. 15 de enero de 2013. <http://www. jstor.org/stable/650819>. 